\title{
Beyond eCK: Perfect Forward Secrecy under Actor Compromise and Ephemeral-Key Reveal
}

\author{
Cas Cremers and Michèle Feltz ${ }^{\star}$ \\ Institute of Information Security \\ ETH Zurich, Switzerland
}

\begin{abstract}
We show that it is possible to achieve perfect forward secrecy in two-message key exchange (KE) protocols that satisfy even stronger security properties than provided by the extended Canetti-Krawczyk (eCK) security model. In particular, we consider perfect forward secrecy in the presence of adversaries that can reveal the long-term secret keys of the actor of a session and reveal ephemeral secret keys.

We propose two new game-based security models for KE protocols. First, we formalize a slightly stronger variant of the eCK security model that we call $\mathrm{eCK}^{w}$. Second, we integrate perfect forward secrecy into $\mathrm{eCK}^{w}$, which gives rise to the even stronger eCK-PFS model. We propose a security-strengthening transformation (i.e., a compiler) between our new models. Given a two-message Diffie-Hellman type protocol secure in $\mathrm{eCK}^{w}$, our transformation yields a two-message protocol that is secure in eCK-PFS. As an example, we show how our transformation can be applied to the NAXOS protocol.
\end{abstract}

Keywords: Key Exchange, Security Models, Protocol Transformations, Perfect Forward Secrecy, Ephemeral-key reveal, Actor compromise.

\section{Introduction}

The majority of recently developed key exchange protocols have been proven secure with respect to game-based security models for key exchange protocols [1, 2, 7, 13, 15. The first such security model was introduced by Bellare and Rogaway 2. In this model, the adversary is modeled as a probabilistic polynomialtime Turing machine that interacts with the protocol participants through queries. The queries specify the capabilities of the adversary. For instance, he can send messages to parties and reveal certain session-keys. The definition of security in the Bellare-Rogaway model requires that (a) two parties who complete matching sessions (i.e., the intended communication partners) compute the same session-key and that (b) the adversary does not learn the sessionkey with more than negligible probability. Building on this work, Canetti and Krawczyk [7] developed a more complex security model that gives the adversary

\footnotetext{
* This work was supported by ETH Research Grant ETH-30 09-3 and the National Competence Center in Research on Mobile Information and Communication Systems (NCCR-MICS), which is supported by the Swiss National Science Foundation.
}

S. Foresti, M. Yung, and F. Martinelli (Eds.): ESORICS 2012, LNCS 7459, pp. 734-751, 2012.

(C) Springer-Verlag Berlin Heidelberg 2012 
additional powers such as access to a session-state query that reveals the internal state of a session. LaMacchia et al. [15] adapted the Canetti-Krawczyk model to capture resilience to key compromise impersonation (KCI) attacks and resilience to the leakage of various combinations of long-term and ephemeral secret keys in a single security model. This model is known as the extended Canetti-Krawczyk (eCK) security model.

One important property of KE protocols that is not guaranteed by the eCK security model is perfect forward secrecy (PFS). This property holds if an adversary cannot learn the session-keys of past sessions, even if he learns the long-term secret keys of all the parties [18]. The designers of the eCK model argued that this property cannot be achieved by two-message KE protocols, based on 13 . In particular, in [13, p. 15], Krawczyk sketched a generic PFS attack, for which he claimed that it breaks the security of any implicitly authenticated two-message $\mathrm{KE}$ protocol. In the attack, the adversary actively interferes with the communication between the parties by injecting self-constructed messages. This enables him to compute the used session-key if he later learns the long-term secret keys of the parties. To prove a slightly weaker notion of forward secrecy for the HMQV protocol, Krawczyk introduced the notion of weak perfect forward secrecy (weakPFS) [13. When the long-term keys are compromised, weak perfect forward secrecy guarantees secrecy of previously established session-keys, but only for sessions in which the adversary did not actively interfere. Krawczyk's comments seem to have led to the incorrect belief that the best that can be achieved for two-message KE protocols is weak perfect forward secrecy [5, 9, 13, 15. As a result, even though the eCK security model [15] guarantees only weak perfect forward secrecy, it is currently described in the literature as the strongest possible security model for two-message KE protocols [8, 15, 17].

Contributions. Our first contribution is to push forward the theoretical limits of key exchange security notions. This contribution has two parts. First, we generalize the eCK security model [15] based on the observation that a restriction on the adversary in the eCK model, whose purpose it is to prevent Krawczyk's PFS attack, is stronger than needed. To weaken this restriction (while still preventing the attack) we introduce the concept of origin-session, which relaxes the notion of matching session. The resulting model, which we call $\mathrm{eCK}^{w}$, specifies a slightly stronger variant of weak perfect forward secrecy than the eCK model. We then integrate perfect forward secrecy into the $\mathrm{eCK}^{w}$ model, which gives rise to the eCK-PFS model. The eCK-PFS model is strictly stronger than $\mathrm{eCK}^{w}$, and also provides more guarantees than independently considering $\mathrm{eCK} / \mathrm{eCK}^{w}$ security and PFS. In particular, security in eCK-PFS implies perfect forward secrecy in the presence of a fully active attacker who can even learn the actor's long-term secret key before the start of the attacked session, or who can learn session-specific ephemeral secret keys (i. e. random coins generated on a per-session basis).

Our second contribution is a generic security-strengthening transformation (a so-called compiler) that contributes towards the modular design approach of KE protocols. Given a two-message Diffie-Hellman (DH) type KE protocol that is secure in $\mathrm{eCK}^{w}$, our transformation yields a two-message protocol that is 
secure in the eCK-PFS model. We show that NAXOS [15], the first key exchange protocol proven secure in the eCK model, is also secure in $\mathrm{eCK}^{w}$ and use our transformation to construct a protocol that is secure in eCK-PFS. Thus, we demonstrate that it is possible for two-message KE protocols to achieve PFS, even under actor compromise (i. e. disclosure of the long-term secret keys of the actor of a session) and leakage of ephemeral secret keys.

Related Work. The majority of related works claim that perfect forward secrecy cannot be achieved in a two-message KE protocol [5, 9, 13, 15. There are two notable exceptions. First, the two-message modified-Okamoto-Tanaka (mOT) protocol by Gennaro et al. 11] provides perfect forward secrecy in the identity-based setting. Additionally, they sketch variants of the protocol for the PKI-based setting. As noted by the authors [11, the mOT protocol and its variants are not resilient against loss of ephemeral keys, and they are therefore insecure in eCKlike models. Second, in [6], Boyd and Gonzalez suggest a transformation $\mathcal{C}$ based on adding MACs on the message exchange of a key-exchange protocol that satisfies weak perfect forward secrecy, to achieve perfect forward secrecy. However, the MAC transformation does not ensure security in eCK-PFS, because it does not guarantee perfect forward secrecy under actor compromise and leakage of ephemeral secret keys. In Section 4 we show that, e.g., $\mathcal{C}($ NAXOS) 6 is insecure in eCK-PFS. The eCK variant for protocols with more than two messages, defined in [14, guarantees perfect forward secrecy. However, this eCK variant cannot be met by any of the protocols from the class we are considering here because it uses the concept of matching session instead of origin-session.

Organization. In Section 2 we recall some standard definitions used in this paper. In Section 3 we motivate and define our security notions $\mathrm{eCK}^{w}$ and eCK-PFS. In Section 4 we provide a transformation that turns any two-message Diffie-Hellman type $\mathrm{KE}$ protocol secure in $\mathrm{eCK}^{w}$ into a two-message $\mathrm{KE}$ protocol secure in eCKPFS. We show how this transformation can be applied to the NAXOS protocol in Section 5. Finally, we conclude in Section 6.

\section{Preliminaries}

Let $G=\langle g\rangle$ be a finite cyclic group of large prime order $p$ with generator $g$.

Definition 1 (GAP-CDH Assumption [19]). The GAP-CDH assumption in $G$ states that, given $g^{u}$ and $g^{v}$, for $u, v$ chosen uniformly at random from $\mathbb{Z}_{p}$, it is computationally infeasible to compute $g^{u v}$ with the help of a decisional Diffie-Hellman (DDH) oracle (that, for any three elements $g^{u}, g^{v}, g^{w} \in G$, replies whether or not $w=u v \bmod p)$.

Definition 2 (Signature Scheme [12]). A signature scheme $\Sigma$ is a tuple of three polynomial-time algorithms (Gen, Sign, Vrfy) satisfying the following:

1. The probabilistic key-generation algorithm Gen takes as input a security parameter $1^{k}$ and outputs a secret/public key pair $(s k, p k)$. 
2. The (possibly probabilistic) signing algorithm Sign takes as input a secret key sk and a message $m \in\{0,1\}^{*}$. It outputs a signature $\sigma:=\operatorname{Sign}_{s k}(m)$.

3. The deterministic verification algorithm Vrfy takes as input a public key pk, a message $m$, and a signature $\sigma$. It outputs a bit $b$, with $b=1$ meaning valid and $b=0$ meaning invalid. We write $b=\operatorname{Vrfy}_{p k}(m, \sigma)$.

Definition 3 (SUF-CMA [4]). A signature scheme $\Sigma=($ Gen, Sign, Vrfy) is strongly existentially unforgeable under an adaptive chosen-message attack if for all probabilistic polynomial-time adversaries $A$, there exists a negligible function negl such that $A d v_{A}^{\text {Sig }}(k) \leq n e g l(k)$, where $A d v_{A}^{\text {Sig }}(k)$ denotes the probability of successfully forging a valid signature $\sigma$ on a message $m$ and $(m, \sigma)$ is not among the pairs $\left(m_{i}, \sigma_{i}\right)(i=1, \ldots, q)$ generated during the query phase to a signature oracle $\mathcal{O}^{\text {Sign }}$ returning a signature for any message $m_{i}$ of the adversary's choice.

\section{$3 \quad$ Key Exchange Security Notions}

We propose two new eCK-like security models for the analysis of key-exchange protocols. The first model called $\mathrm{eCK}^{w}$ captures a slightly stronger form of weakPFS than the eCK model. The second model called eCK-PFS integrates PFS directly into $\mathrm{eCK}^{w}$.

\subsection{Motivation for the New Models}

eCK $^{w}$ : Strengthening Weak-PFS. As stated in the introduction, the eCK model captures weak perfect forward secrecy but not perfect forward secrecy, based on Krawczyk's generic PFS attack [13. We briefly recall the attack. Consider a two-message protocol in which the agents exchange ephemeral public Diffie-Hellman keys, i. e., $g^{x}$ and $g^{y}$, where $x$ and $y$ are chosen at random from $\mathbb{Z}_{p}$ (for some large prime $p$ ). The adversary, impersonating party $\hat{A}$, generates a random value $x\left(\in \mathbb{Z}_{p}\right)$ and sends $g^{x}$ to a responder session at party $\hat{B}$. $\hat{B}$ responds by sending $g^{y}$ and computes the session key. The adversary chooses $\hat{B}$ 's session as the test-session, i. e. the session under attack, and reveals $\hat{A}$ 's long-term secret key after $\hat{B}$ 's session ends. Now the adversary can simply follow all protocol steps that an honest party $\hat{A}$ would have performed using $x$ and $\hat{A}$ 's long-term secret key. In particular, the adversary can compute the same session-key as the test-session, violating PFS.

Krawczyk's attack works directly for all two-message KE protocols that exchange DH keys of the form $g^{z}$, where $z$ does not involve the sender's long-term secret key, such as HMQV 13. Additionally, the attack also works on protocols like NAXOS [15, where $z$ is a hash of the sender's long-term secret key and a random value. The adversary can just replace this value by an arbitrary value.

To still prove some form of forward secrecy for such protocols, Krawczyk introduced the notion of weak-PFS. In weak-PFS, the adversary is not allowed to actively interfere with the messages exchanged by the test-session. This prevents the attack because the adversary is no longer allowed to insert his own $\mathrm{DH}$ 
exponential. Similarly, in the eCK model, this restriction on interfering with the test-session is modeled by checking if a matching session exists [15, p. 5]. If this is the case, then the adversary must have been passive and he is allowed to reveal the long-term secret keys of the actor and the intended communication partner of a session. If there is no matching session, the adversary is not allowed to reveal the long-term secret key of the intended communication partner.

We observe that Krawczyk's attack only depends on the adversary injecting or modifying the message received by the test-session; he does not need to actively interfere with the message sent by the test-session. However, eCK models passivity of the adversary in the test-session by checking whether a matching session for the test-session exists, which also prevents the adversary from modifying (or deleting) the message sent by the test-session. In this sense, the restriction on the adversary in eCK is sufficient but not necessary for the prevention of Krawczyk's attack. We therefore relax the notion of matching sessions and introduce the concept of origin-session. This allows us to capture the adversary's capability of revealing the long-term secret key of the intended communication partner (i. e. the peer) of the test-session $s$ in case an origin-session $s^{\prime}$ for $s$ exists even though no session matching to $s$ exists. Thus, in contrast to the eCK model, the adversary may reveal the long-term key of the peer of the test-session $s$ in case an origin-session $s^{\prime}$ for session $s$ exists and

- actively interfere with the message sent by the test-session (e. g. by modifying it or injecting his own message), or

- replay a message from another session to the test-session (as in [6]), or

- leave session $s^{\prime}$ incomplete (in case session $s^{\prime}$ is in the initiator role).

We call our strengthened variant of the eCK model the $\mathrm{eCK}^{w}$ model.

eCK-PFS: Integrating PFS into $\mathbf{e C K}^{w}$. We extend the $\mathrm{eCK}^{w}$ model by integrating perfect forward secrecy which yields the strictly stronger eCK-PFS model. Perfect forward secrecy is reflected in eCK-PFS by allowing the adversary to reveal the long-term secret keys of all the protocol participants after the end of the test-session. These keys can be revealed irrespective of the existence of an origin-session (or a matching session). This attack scenario is neither captured in $\mathrm{eCK}^{w}$ (nor in eCK or [6]) if the origin-session (matching session) does not exist for the test-session.

\subsection{Defining eCK ${ }^{w}$ and eCK-PFS}

Terminology. Let $\mathcal{P}=\left\{\hat{P}_{1}, \hat{P}_{2}, \ldots, \hat{P}_{N}\right\}$ be a finite set of $N$ parties' identities. Each party can execute multiple instances of a $\mathrm{KE}$ protocol, called sessions, concurrently. We denote session $i$ at party $\hat{P}$ as the tuple $(\hat{P}, i) \in \mathcal{P} \times$ $\mathbb{N}$. We associate to each session $s \in \mathcal{P} \times \mathbb{N}$ a quintuple of variables $T_{s}=$ $\left(s_{\text {actor }}, s_{\text {peer }}, s_{\text {role }}, s_{\text {sent }}, s_{\text {recv }}\right) \in \mathcal{P}^{2} \times\{\mathcal{I}, \mathcal{R}\} \times\left(\{0,1\}^{*} \cup\{-\}\right)^{2}$. The variables $s_{\text {actor }}, s_{\text {peer }}$ denote the identities of the actor and intended peer of session $s, s_{\text {role }}$ denotes the role that the session is executing (either initiator or responder), and $s_{\text {sent }}, s_{\text {recv }}$ denote the concatenation of timely ordered messages as sent/received 
by $s_{\text {actor }}$ during session $s$, where " - "denotes a special symbol not in $\{0,1\}$ that represents the empty sequence. The values of the variables $s_{\text {peer }}$ and $s_{\text {role }}$ are set upon activation of session $s$ and the values of the variables $s_{\text {sent }}$ and $s_{\text {recv }}$ are defined by the protocol execution steps. A session can only be activated once.

The notion of matching sessions specifies when two sessions are supposed to be intended communication partners. Here we formalize the matching sessions definition from the eCK model [15] which is based on matching conversations.

Definition 4 (matching sessions). Two completed sessions $s$ and $s^{\prime}$ are said to be matching if

$$
s_{\text {actor }}=s_{\text {peer }}^{\prime} \wedge s_{\text {peer }}=s_{\text {actor }}^{\prime} \wedge s_{\text {sent }}=s_{\text {recv }}^{\prime} \wedge s_{\text {recv }}=s_{\text {sent }}^{\prime} \wedge s_{\text {role }} \neq s_{\text {role }}^{\prime} .
$$

To relate a message received (and accepted) by some session to the session it originates from (if the latter exists), we introduce the concept of origin-session. If an origin-session $s^{\prime}$ for some session $s$ exists, then the messages received by session $s$ have not been modified or injected (as in Krawczyk's PFS attack [13]) by the adversary.

Definition 5 (origin-session). We say that a (possibly incomplete) session $s^{\prime}$ is an origin-session for a completed session $s$ when $s_{\text {sent }}^{\prime}=s_{\text {recv }}$.

Note that, if two completed sessions $s, s^{\prime}$ are matching, then $s$ and $s^{\prime}$ are originsessions for each other. However, if session $s$ is an origin-session for some session $s^{\prime}$, then it might not necessarily be a matching session for $s^{\prime}$ (e. g. in case the roles of the sessions are identical). Thus, a session being a matching session for some session is a stronger requirement than a session being an origin-session for some session.

Adversarial capabilities. Similar to the eCK model [15], we model the adversary as a probabilistic polynomial-time (PPT) Turing machine that controls all communications between parties through the following queries:

1. $\operatorname{send}(s, v)$. This query models the adversary sending message $v$ to session $s$. The adversary is given the response generated by the session according to the protocol. The variables $s_{\text {sent }}$ and $s_{\text {recv }}$ are updated accordingly (by concatenation). Abusing notation, we allow the adversary to activate an initiator session with peer $\hat{Q}$, via a send $(s, \hat{Q})$ query and a responder session by sending a message $m$ to session $s$ on behalf of $\hat{Q}$, via a send $(s, \hat{Q}, m)$ query. In these cases, $s_{\text {peer }}$ is set to $\hat{Q}$ and $s_{\text {role }}$ is set to $\mathcal{I}$ and $\mathcal{R}$, respectively. The adversary is given the session's response according to the protocol and the variables $s_{\text {sent }}, s_{\text {recv }}$ are initialized accordingly.

2. corrupt $(\hat{P})$. This query reveals the long-term keys of party $\hat{P}$.

3. ephemeral-key $(s)$. This query reveals the ephemeral secret keys (i.e., the random coins) of session $s$.

4. session-key $(s)$. This query returns the session key for a completed session $s$ (i. e. a session that has accepted/computed a session-key). 
5. test-session $(s)$. To respond to this query, a random bit $b$ is chosen. If $b=0$, then the session-key established in session $s$ is returned. Otherwise, a random key is returned according to the probability distribution of keys generated by the protocol. This query can only be issued to a completed session.

Notions of Freshness. An adversary that can perform the above queries can simply reveal the session key of all sessions, breaking any protocol. The intuition underlying Bellare-Rogaway style KE models is to put minimal restrictions on the adversary with respect to performing these queries, such that there still exist protocols that are secure in the presence of such an adversary. The restrictions on the queries made by the adversary are formalized by the notion of fresh sessions.

Definition 6 (Fresh session in $\mathbf{e C K}^{w}$ ). A completed session $s$ in security experiment $W$ is said to be fresh in $e C K^{w}$ if all of the following conditions hold:

1. W does not include the query session-key $(s)$,

2. for all sessions $s^{*}$ such that $s^{*}$ matches $s, W$ does not include session-key $\left(s^{*}\right)$,

3. $W$ does not include both $\operatorname{corrupt}\left(s_{\text {actor }}\right)$ and ephemeral-key $(s)$,

4. for all sessions $s^{\prime}$ such that $s^{\prime}$ is an origin-session for session $s, W$ does not include both corrupt $\left(s_{\text {peer }}\right)$ and ephemeral-key $\left(s^{\prime}\right)$, and

5. if there exists no origin-session for session $s$, then $W$ does not include a corrupt $\left(s_{\text {peer }}\right)$ query.

Definition 7 (Fresh session in eCK-PFS). A completed session $s$ in experiment $W$ is said to be fresh in eCK-PFS if all of the following conditions hold:

1. $W$ does not include the query session-key $(s)$,

2. for all sessions $s^{*}$ such that $s^{*}$ matches $s, W$ does not include session-key $\left(s^{*}\right)$,

3. $W$ does not include both corrupt $\left(s_{\text {actor }}\right)$ and ephemeral-key $(s)$,

4. for all sessions $s^{\prime}$ such that $s^{\prime}$ is an origin-session for session $s, W$ does not include both corrupt $\left(s_{\text {peer }}\right)$ and ephemeral-key $\left(s^{\prime}\right)$, and

5. if there exists no origin-session for session $s$, then $W$ does not include a corrupt $\left(s_{\text {peer }}\right)$ query before the completion of session $s$.

Security Experiment $W$ in model $M$. Security of a key-exchange protocol $\Pi$ is defined via a security experiment $W$ (or attack game) played by an adversary $E$, modeled as a PPT algorithm, against a challenger. Before the experiment starts, each party $\hat{P}$ runs a key-generation algorithm that takes as input a security parameter $1^{k}$ and outputs valid static secret/public key pair(s). The public key(s) of each party are distributed in an authenticated way to all other parties. The adversary $E$ is given access to all public data. The setting of the security experiment $W$ can be described in four successive stages, as follows:

1. The adversary $E$ can perform send, corrupt, ephemeral-key, and session-key queries.

2. At some point in the experiment, $E$ issues a test-session query to a completed session that is fresh in model $M$ by the time the query is issued. The challenger chooses a random bit $b$ and provides $E$ with either the real session-key of the test-session (for $b=0$ ) or a random key from the key space (for $b=1$ ). 
3. The adversary may continue with send, corrupt, ephemeral-key and session-key queries, without rendering the test-session un-fresh in model $M$.

4. Finally, $E$ outputs a bit $b^{\prime}$ as his guess for $b$.

The adversary $E$ wins the security experiment $W$ if he correctly guesses the bit $b$ chosen by the challenger during the test-session query (i. e. if $b=b^{\prime}$ where $b^{\prime}$ denotes $E$ 's guess). Success of $E$ in the experiment is expressed in terms of $E$ 's advantage in distinguishing whether he received the real or a random session-key in response to the test-session query. The advantage of adversary $E$ in the above security experiment against a key exchange protocol $\Pi$ for security parameter $k$ is defined as $A d v_{E}^{\Pi}(k)=\left|2 P\left(b=b^{\prime}\right)-1\right|$.

Definition 8. A key exchange protocol $\Pi$ is said to be secure in model $M \in$ $\left\{e C K^{w}, e C K-P F S\right\}$ if, for all PPT adversaries E, it holds that

- if two parties successfully complete matching sessions, then they compute the same session key, and

- E has no more than a negligible advantage in winning security experiment $W$ in model $M$, that is, there exists a negligible function negl in the security parameter $k$ such that $A d v_{E}^{\Pi}(k) \leq n e g l(k)$.

Comparison between $e C K^{w}$ and $e C K-P F S$. The eCK-PFS model is strictly stronger than $\mathrm{eCK}^{w}$ because it captures more attack scenarios. The eCK-PFS model allows the adversary to corrupt all parties after the test-session is completed (regardless of whether an origin-session exists for the test-session), capturing perfect forward secrecy. In contrast, in case there is no origin-session for the test-session, the adversary is not allowed to reveal the long-term secret key of the peer of the test-session in the $\mathrm{eCK}^{w}$ model. As an example, NAXOS is provably secure in $\mathrm{eCK}^{w}$, as we show in Section 5, but insecure in eCK-PFS due to the PFS attack described in Subsection 3.1 .

\section{A Transformation from $\mathrm{eCK}^{w}$ to eCK-PFS}

We define a class of two-message Diffie-Hellman type key exchange protocols (similar to the class of KE protocols in [6]). Then, we present a securitystrengthening transformation (compiler) that can be applied to any such protocol. Finally we show that this transformation turns any KE protocol secure in $\mathrm{eCK}^{w}$ into a KE protocol secure in eCK-PFS.

Let $k$ be a security parameter and let $G$ be a finite cyclic group of prime order $p$ with generator $g$, where $p=O\left(2^{k}\right)$. Let $\Omega$ be static publicly known data such as parties' identities, their long-term public keys or publicly known functions and parameters. Let $S$ be a set of constants from which random values are chosen (e.g. $S=\mathbb{Z}_{p}$ or $S=\{0,1\}^{k}$ ). We denote by $x \in_{R} S$ that $x$ is chosen uniformly at random from the set $S$. In the generic two-message DH type protocol, illustrated in Figure 1, party $\hat{A}$ 's long-term secret key is $a \in_{R} \mathbb{Z}_{p}$ and $\hat{A}$ 's long-term public key is $A=g^{a}$. The session-specific ephemeral secret key of the session at party 

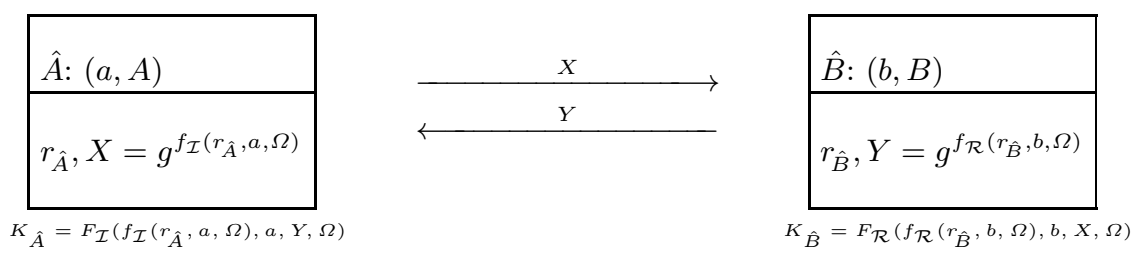

Fig. 1. A generic two-message DH type protocol

$\hat{A}$ is denoted by $r_{\hat{A}} \in_{R} S$ and the corresponding ephemeral public key is denoted by $X$. Similarly, party $\hat{B}$ 's long-term secret/public key pair is $(b, B)$ and the ephemeral secret/public key pair of the session at $\hat{B}$ is denoted by $\left(r_{\hat{B}}, Y\right)$. The public functions $f_{\mathcal{I}}, f_{\mathcal{R}}:\{0,1\}^{*} \rightarrow \mathbb{Z}_{p}$ depend on the ephemeral secret key and may depend on the long-term secret key or on public information. The public functions $F_{\mathcal{I}}, F_{\mathcal{R}}:\{0,1\}^{*} \rightarrow\{0,1\}^{k}$ depend on the Diffie-Hellman exponent the long-term secret key, the received Diffie-Hellman exponential and other public information. We assume that the public keys of all parties are known to all other participants in the protocol.

Protocol description. The generic two-message DH type protocol, depicted in Figure 1, proceeds as follows:

1. Upon activation of session $s=(\hat{A}, i) \in \mathcal{P} \times \mathbb{N}$ with peer $\hat{B}, \hat{A}$ (the initiator) performs the steps:

- Choose an ephemeral secret key $r_{\hat{A}} \in_{R} S$ and compute $X=g^{f_{\mathcal{I}}\left(r_{\hat{A}}, a, \Omega\right)}$.

- Send $X$ (and possibly other public data, e. g. identities of peer and actor of the session) to $\hat{B}$.

- Initialize $T_{s}$ to $(\hat{A}, \hat{B}, \mathcal{I}, m,-)$, where $m$ denotes the message sent by session $s$.

2. Upon activation of session $s^{\prime}=(\hat{B}, j) \in \mathcal{P} \times \mathbb{N}$ with message $X$ (and possibly other data) on behalf of $\hat{A}$, party $\hat{B}$ (the responder) performs the steps:

- Check that $X \in G$.

- Choose an ephemeral secret key $r_{\hat{B}} \in_{R} S$ and compute $Y=g^{f_{\mathcal{R}}\left(r_{\hat{B}}, b, \Omega\right)}$.

- Compute $K_{\hat{B}}=F_{\mathcal{R}}\left(f_{\mathcal{R}}\left(r_{\hat{B}}, b, \Omega\right), b, X, \Omega\right)$.

- Send $Y$ (and possibly other public data) to $\hat{A}$.

- Set $T_{s^{\prime}}$ to $\left(\hat{B}, \hat{A}, \mathcal{R}, m^{\prime}, n^{\prime}\right)$, where $m^{\prime}$ denotes the message sent by session $s^{\prime}$ and $n^{\prime}$ the message received by session $s^{\prime}$, and complete the session by accepting $K_{\hat{B}}$ as the session-key.

3. Upon receiving message $Y$ (with possibly other data) in session $s$, party $\hat{A}$ performs the steps:

- Check that $Y \in G$.

- Compute $K_{\hat{A}}=F_{\mathcal{I}}\left(f_{\mathcal{I}}\left(r_{\hat{A}}, a, \Omega\right), a, Y, \Omega\right)$.

- Update $T_{s}$ to $(\hat{A}, \hat{B}, \mathcal{I}, m, n)$ and complete the session by accepting $K_{\hat{A}}$ as the session-key. 
The above description also applies to protocols with additional checks, which we omit for clarity. We assume that whenever a check in a session fails, all sessionspecific data is erased from memory and the session is aborted, i. e., it terminates without establishing a session-key.

Definition 9 (Protocol Class $\mathcal{D H}-2)$. We define $\mathcal{D H}-2$ as the class of all two-message key-exchange protocols that follow the description of a generic DH type protocol and meet the following validity requirement:

- in the presence of an eavesdropping adversary, two parties $\hat{A}$ and $\hat{B}$ can complete matching sessions (in the sense of Definition 4), in which case they hold the same session-key.

The validity requirement requires that if the messages of two parties $\hat{A}$ and $\hat{B}$ are faithfully relayed to each other, then both parties end up with a shared session-key (see also [1]). Note that, e.g., the KE protocols NAXOS [15], NAXOS+ [17, NETS [16] and CMQV [21] belong to the class $\mathcal{D H}-2$.

Protocol transformation. We now show how to transform any protocol $\Pi \in$ $\mathcal{D H}-2$ into a two-message protocol $\operatorname{SIG}(\Pi)$, shown in Figure 2, by applying the signature transformation SIG. Party $\hat{A}$ has two independent valid long-term secret/public key pairs, one pair $(a, A)$ from protocol $\Pi$ and one pair $\left(s k_{\hat{A}}, p k_{\hat{A}}\right)$ for use in a digital signature scheme $\Sigma$ with security parameter $k$. Similarly, party $\hat{B}$ 's long-term secret/public key pairs are $(b, B)$ and $\left(s k_{\hat{B}}, p k_{\hat{B}}\right)$. The transformed protocol SIG $(\Pi)$ in Figure 2 proceeds as protocol $\Pi$ except that each party needs to additionally sign a message using its secret signature key and check that the received signature on a message is valid with respect to the long-term public key of its peer. The fields between square brackets within the signature are optional.

Security analysis. We show in Theorem 1 below that the SIG transformation is a security-strengthening transformation from the $\mathrm{eCK}^{w}$ model to the stronger model eCK-PFS provided that the digital signature scheme is strongly existentially unforgeable under an adaptive chosen-message attack (SUF-CMA) as well as deterministic. For certain randomized signature schemes, an efficient adversary can compute the secret (signature) key given the corresponding public key, a signature on any message using the secret key, and the random coins involved in the signature generation learned through an ephemeral-key query (as noted in [15]).

$$
\begin{aligned}
& \hat{A}:(a, A),\left(s k_{\hat{A}}, p k_{\hat{A}}\right) \\
& \hline r_{\hat{A}}, X=g^{f_{\mathcal{I}}\left(r_{\hat{A}}, a, \Omega\right)} \\
& K_{\hat{A}}=F_{\mathcal{I}}\left(f_{\mathcal{I}}\left(r_{\hat{A}}, a, \Omega\right), a, Y, \Omega\right)
\end{aligned}
$$
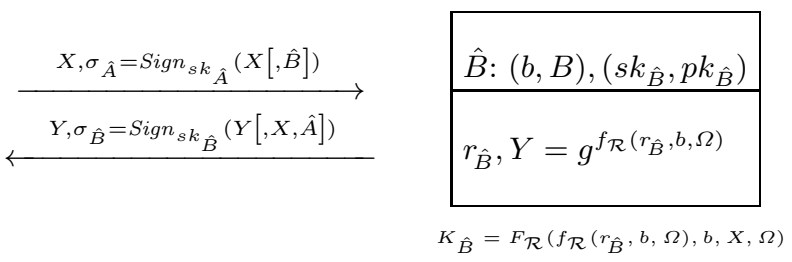

Fig. 2. A transformed generic protocol $\operatorname{SIG}(\Pi)$ 
The following lemma is used in the proof of Theorem 1 .

Lemma 1 (Difference Lemma [20]). Let $A, B, F$ be events defined on some probability space. Suppose that event $A \wedge F^{c}$ occurs if and only if event $B \wedge F^{c}$ occurs. Then $|P(A)-P(B)| \leq P(F)$.

Theorem 1. Let $\Pi \in \mathcal{D H}$-2 be a protocol secure in the eCK ${ }^{w}$ model. Under the assumption that the signature scheme is deterministic and SUF-CMA, the protocol $\mathrm{SIG}(\Pi)$ is a secure key-exchange protocol in the eCK-PFS model.

Proof. It is straightforward to verify the first condition of Definition 8, i. e., that matching sessions of protocol $\mathrm{SIG}(\Pi)$ compute the same key (since matching sessions of protocol $\Pi$ compute the same key). We show next that the second condition of Definition 8 holds, i. e., the adversary has no more than a negligible advantage in distinguishing the session key from a random key. We present a security proof structured as a sequence of games, a proof technique introduced in [20]. Let $S_{i}$ denote the event that the adversary correctly guesses the bit chosen by the challenger to answer the test-session query in Game $i$ and let $\alpha_{i}=\left|2 P\left(S_{i}\right)-1\right|$ denote the advantage of the adversary in Game $i$. Let $N, q_{s}$ be upper bounds on the number of parties and activated sessions, respectively.

Game 0. This game reflects the security experiment $W$ in model eCK-PFS, as defined in Subsection 3.2, played by a PPT adversary $E$ against the protocol $\operatorname{SIG}(\Pi)$.

Game 1. [Transition based on a small failure event] Let $\operatorname{Coll}_{\mathrm{SIG}(\Pi)}$ be the small failure event that a collision for protocol SIG $(\Pi)$ occurs (e.g. in ephemeral secret keys). As soon as event $\operatorname{Coll}_{\mathrm{SIG}(\Pi)}$ occurs, the attack game stops.

Analysis of Game 1. Game 0 is identical to Game 1 up to the point in the experiment where event $\operatorname{Coll}_{\mathrm{SIG}(\Pi)}$ occurs for the first time. The Difference Lemma yields that $\left|P\left(S_{0}\right)-P\left(S_{1}\right)\right| \leq P\left(\operatorname{Coll}_{\mathrm{SIG}(\Pi)}\right)$. Hence,

$$
\begin{aligned}
\alpha_{0} & =\left|2 P\left(S_{0}\right)-1\right|=2\left|P\left(S_{0}\right)-P\left(S_{1}\right)+P\left(S_{1}\right)-1 / 2\right| \\
& \leq 2\left(\left|P\left(S_{0}\right)-P\left(S_{1}\right)\right|+\left|P\left(S_{1}\right)-1 / 2\right|\right) \\
& \leq 2 P\left(\text { Coll }_{\mathrm{SIG}(\Pi)}\right)+\alpha_{1} .
\end{aligned}
$$

Game 2. [Transition based on a large failure event (see [5, 10])] Before the adversary $E$ starts the attack game, the challenger chooses a random value $m \in_{R}$ $\left\{1,2, \ldots, q_{s}\right\}$. The $m$-th session activated by $E$, denoted by $s^{*}$, is the session on which the challenger wants the adversary to be tested. Let $T$ be the event that the test-session is not session $s^{*}$. If event $T$ occurs, then the attack game halts and the adversary outputs a random bit. 
Analysis of Game 2. Event $T$ is non-negligible, the environment can efficiently detect it and $T$ is independent of the output in Game 1 (i. e. $P\left(S_{1} \mid T\right)=P\left(S_{1}\right)$ ). If $T$ does not occur, then the attacker $E$ will output the same bit in Game 2 as it did in Game 1 (so that $P\left(S_{2} \mid T^{c}\right)=P\left(S_{1} \mid T^{c}\right)=P\left(S_{1}\right)$ ). If event $T$ occurs in Game 2 , then the attack game halts and the adversary $E$ outputs a random bit (so that $\left.P\left(S_{2} \mid T\right)=1 / 2\right)$. We have,

$$
\begin{aligned}
P\left(S_{2}\right) & =P\left(S_{2} \mid T\right) P(T)+P\left(S_{2} \mid T^{c}\right) P\left(T^{c}\right)=\frac{1}{2} P(T)+P\left(S_{1}\right) P\left(T^{c}\right) \\
& =P\left(T^{c}\right)\left(P\left(S_{1}\right)-\frac{1}{2}\right)+\frac{1}{2}
\end{aligned}
$$

Hence we get, $\alpha_{2}=\left|2 P\left(S_{2}\right)-1\right|=P\left(T^{c}\right)\left|2 P\left(S_{1}\right)-1\right|=\frac{1}{q_{s}} \alpha_{1}$.

Suppose w.l.o.g. that $s_{\text {role }}^{*}=\mathcal{I}$ and that protocol $\Pi$ does not include optional public information in the sent messages. Let $F$ be a forgery event with respect to the long-term public key $p k_{\hat{P}}$ of party $\hat{P}$, that is, adversary $E$ issues a send $\left(s^{*}, V, \sigma\right)$ query to session $s^{*}$ being incomplete such that

- $\sigma$ is a valid signature on message $m=\left(V,\left[W, s_{\text {actor }}^{*}\right]\right)$ with respect to the public key of $\hat{P}$, where $W$ is the Diffie-Hellman exponential contained in message $s_{\text {sent }}^{*}$, and

- $(V, \sigma)$ has never been output by party $\hat{P}$ in response to a send query.

Game 3. [Transition based on a small failure event] This game is the same as the previous one except that when a forgery event $F$ with respect to the long-term public key of some party $\hat{P} \in \mathcal{P}$ occurs, the experiment halts and $E$ outputs a random bit.

Analysis of Game 3. The analysis of Game 3 proceeds in several steps.

Consider first the following two cases.

1. If $E$ issues a corrupt $(\hat{P})$ query before the completion of session $s^{*}$, then this query would render session $s^{*}$ un-fresh. This would have caused Game 2 to abort since session $s^{*}$ would not be the test-session. Recall that the test-session query can only be issued to a session that is fresh by the time the query is issued. Hence this case can be excluded.

2 . If $E$ does not issue a corrupt $(\hat{P})$ query before the completion of session $s^{*}$, then he can only impersonate party $\hat{P}$ to session $s^{*}$ by forging a signature on a message with respect to the long-term public key of $\hat{P}$.

Claim. We have $\left|P\left(S_{2}\right)-P\left(S_{3}\right)\right| \leq P(F)$.

Proof. If event $F$ does not occur, then Game 2 and 3 proceed identically (i.e. $\left.S_{2} \wedge F^{c} \Leftrightarrow S_{3} \wedge F^{c}\right)$. The Difference Lemma yields that $\left|P\left(S_{2}\right)-P\left(S_{3}\right)\right| \leq P(F)$.

Claim. If the deterministic signature scheme is SUF-CMA, then $P(F)$ is negligible. More precisely, $P(F) \leq N A d v_{M}^{S i g n}(k)$, where $A d v_{M}^{S i g n}(k)$ denotes the probability of a successful forgery. 
Proof. Consider the following algorithm $M$ using adversary $E$ as a subroutine. $M$ is given a public signature key $p k$ and access to the corresponding signature oracle $\mathcal{O}^{\text {Sign }}$. It selects at random one of the $N$ parties and sets its public key to $p k$. We denote this party by $\hat{P}$ and its signature key pair by $\left(s k_{\hat{P}}, p k_{\hat{P}}\right)$. Further, the algorithm $M$ chooses signature key pairs $\left(s k_{i}, p k_{i}\right)$ for all parties $\hat{P}_{i} \in \mathcal{P}$ with $\hat{P}_{i} \neq \hat{P}$ and stores the associated secret keys. It also chooses key pairs $\left(c_{i}, C_{i}\right)$ for all parties $\hat{P}_{i} \in \mathcal{P}$ as needed for protocol $\Pi$ and stores the associated secret keys.

\section{ALGORITHM $M$ :}

1. Run $E$ on input $1^{k}$ and the public keys for all of the $N$ parties.

2. If $E$ issues a send $(z, \hat{Q})$ query to activate session $z$ with peer $\hat{Q} \in \mathcal{P}$, then answer it as follows.

- If $z_{\text {actor }} \neq \hat{P}$, then choose $x \in_{R} \mathbb{Z}_{p}$ to get $X=g^{x}$, compute the signature $\sigma$ on message $m=(X[, \hat{Q}])$ on behalf of $z_{\text {actor }}$ and return the message $(X, \sigma)$ to $E$.

- If $z_{\text {actor }}=\hat{P}$, then choose $x \in_{R} \mathbb{Z}_{p}$ to get $X=g^{x}$ and query the signature oracle on message $m=(X[, \hat{Q}])$ which returns the signature $\sigma$ on message $m$. Store the pair $(m, \sigma)$ in a table $L$, initially empty, and return the message $(X, \sigma)$ to $E$.

3. If $E$ issues a send $(z, \hat{Q}, m)$ query to activate session $z$, then answer it as follows. First check whether message $m$ is of the form $(X, \sigma)$ for some $X \in G$ and $\sigma$ a valid signature on message $\left(X\left[, z_{\text {actor }}\right]\right)$ with respect to the public key of $\hat{Q}$. If the checks succeed, then:

- If $z_{\text {actor }} \neq \hat{P}$, then choose $y \in_{R} \mathbb{Z}_{p}$ to get $Y=g^{y}$, compute the signature $\sigma$ on message $m=(Y[, X, \hat{Q}])$ on behalf of $z_{\text {actor }}$ and return the message $(Y, \sigma)$ to $E$.

- If $z_{\text {actor }}=\hat{P}$, then choose $y \in_{R} \mathbb{Z}_{p}$ to get $Y=g^{x}$ and query the signature oracle on message $m=(Y[, X, \hat{Q}])$ which returns the signature $\sigma$ on message $m$. Store the pair $(m, \sigma)$ in table $L$ (initially empty) and return the message $(Y, \sigma)$ to $E$.

If one of the checks does not succeed, then abort session $z$.

4. If $E$ issues a send $(z, m)$ query to session $z$ in role $\mathcal{I}$, then check whether message $m$ is of the form $(Y, \sigma)$ for some $Y \in G$ and $\sigma$ a valid signature on message $\left(Y\left[, X, z_{\text {actor }}\right]\right)$ with respect to the public key of $z_{\text {peer }}$ (where $W \in G$ is contained in message $s_{\text {sent }}^{*}$ ). If the check fails, then abort session $z$.

5. If $E$ makes a send $\left(s^{*}, V, \sigma\right)$ query, where $\sigma$ is a valid signature with respect to the public key $p k_{\hat{P}}$ of party $\hat{P}$ on message $m=\left(V\left[, W, s_{\text {actor }}^{*}\right]\right.$ ) (where $W \in G$ is contained in $s_{\text {sent }}^{*}$ ), before the completion of the test-session $s^{*}$ and $(m, \sigma) \notin L$, then stop $E$ and output $(m, \sigma)$ as a forgery.

6. The queries session-key, ephemeral-key are answered in the appropriate way since $M$ has chosen the ephemeral secret keys for all the sessions and the long-term secret keys for use in protocol $\Pi$ for all the parties.

7. The queries corrupt $\left(\hat{P}_{i}\right)$, where $\hat{P}_{i} \in \mathcal{P}$ and $\hat{P}_{i} \neq \hat{P}$, are answered in the appropriate way since $M$ knows the secret key pairs of the parties $\hat{P}_{i} \neq \hat{P}$.

8. If $E$ issues the query test-session $\left(s^{*}\right)$, then abort with failure. 
Under event $F$, algorithm $M$ is successful as described in Step 5 and the abortion as in Step 8 does not occur. The probability that $E$ succeeds in forging a signature with respect to the public key of $\hat{P}$ is bounded above by the probability that $M$ outputs a forgery multiplied by the number of parties, that is, $P(F) \leq N A d v_{M}^{\text {Sign }}(k)$.

Claim. Let $A d v_{E}^{\mathrm{SIG}(\Pi) \text {, Game } 3, O}(k):=\left|2 P\left(S_{3} \mid O\right)-1\right|$, where $O$ denotes the event that there is an origin-session for the test-session. It holds that $A d v_{E}^{\mathrm{SIG}(\Pi), \text { Game } 3}$ $(k)=\max \left(0, A d v_{E}^{\mathrm{SIG}(\Pi), \text { Game } 3, O}(k)\right)$.

Proof. Note that $\left|2 P\left(S_{3} \mid F\right)-1\right|=\left|2 \frac{1}{2}-1\right|=0$ (since, when event $F$ occurs in Game 3, $E$ outputs a random bit) and that if event $F$ does not occur, then there exists an origin-session for the test-session.

We next establish an upper bound for $A d v_{E}^{\mathrm{SIG}(\Pi) \text {, Game } 3, O}(k)$ in terms of the security of protocol $\Pi$.

Claim. Assume that in Game 3 there exists a unique1 origin-session $s$ for the test-session $s^{*}$ with $s_{\text {actor }}=s_{\text {peer }}^{*}$. If there is an efficient adversary $E$ in eCK-PFS succeeding in Game 3 against protocol SIG $(\Pi)$ with non-negligible advantage, then we can construct an efficient adversary $E^{\prime}$ in $\mathrm{eCK}^{w}$ succeeding in Game 3 against protocol $\Pi$ with non-negligible advantage using adversary $E$ as a subroutine. Moreover, it holds that $A d v_{E}^{\mathrm{SIG}(\Pi) \text {, Game } 3, O}(k) \leq A d v_{E^{\prime}}^{\Pi \text {, Game } 3, O}(k)$.

Proof. Fix an efficient adversary $E$ in eCK-PFS succeeding in Game 3 against protocol $\mathrm{SIG}(\Pi)$ with non-negligible advantage. Let us construct an adversary $E^{\prime}$ in $\mathrm{eCK}^{w}$ succeeding in Game 3 against protocol $\Pi$ with non-negligible advantage using adversary $E$ as a subroutine.

ALGORITHM $E^{\prime}: E^{\prime}$ chooses secret/public signature key pairs for all the parties and stores the associated secret signature keys. It is given all public knowledge, such as public (non-signature) keys for all the parties.

1. Run $E$ against $\operatorname{SIG}(\Pi)$ on input $1^{k}$ and the public key pairs for all of the $N$ parties.

2. When $E$ issues a corrupt $(\hat{P})$ query to some party $\hat{P}, E^{\prime}$ issues that query to party $\hat{P}$ and returns the answer to that query together with the secret signature key of $\hat{P}$ (that $E^{\prime}$ has chosen) to $E$.

3. When $E$ issues an ephemeral-key or a session-key query to some session $z, E^{\prime}$ issues that query to session $z$ and returns the answer to $E$.

4. send queries are answered in the following way.

- If $E$ issues a send $(z, \hat{Q})$ query to activate session $z$ with peer $\hat{Q}$, then $E^{\prime}$ issues the same query to session $z$. The response is a message $W(\in G)$. Since $E^{\prime}$ knows the secret signature key of $z_{\text {actor }}$, it can sign the message

${ }^{1}$ No collision in the ephemeral secret keys occurs for $\operatorname{SIG}(\Pi)$ (where $\Pi \in \mathcal{D H}-2$ ) since otherwise Game 1 would have caused the game to abort. 
$m=(W[, \hat{Q}])$ on its behalf and then return the message $(W, \sigma)$ to $E$, where $\sigma$ denotes the signature on $m$ with respect to the public key of $z_{\text {actor }}$.

- If $E$ issues a send $(z, \hat{Q}, m)$ query to activate session $z$, where message $m$ is of the form $(W, \sigma)$, then $E^{\prime}$ first checks whether $W \in G$ and second whether $\sigma$ is a valid signature on message $\left(W\left[, z_{\text {actor }}\right]\right)$ with respect to the public key of $\hat{Q}$. If the checks succeed, then $E^{\prime}$ issues the query $\operatorname{send}(z, W)$ to session $z$. The response is a message $V \in G$. Since $E^{\prime}$ knows the secret signature key of $z_{\text {actor }}$, it can sign the message $m=(V[, W, \hat{Q}])$ on its behalf and then return the message $(V, \sigma)$ to $E$, where $\sigma$ denotes the signature on $m$ with respect to the public key of $z_{\text {actor }}$.

- If $E$ issues a send $(z, m)$ query, where message $m$ is of the form $(V, \sigma)$, then $E^{\prime}$ first checks whether $V \in G$ and second whether $\sigma$ is a valid signature on message $\left(V\left[, W, z_{\text {actor }}\right]\right)$ with respect to the public key of $z_{\text {peer }}$, where $W$ is the Diffie-Hellman exponential contained in $z_{\text {sent }}$. If the checks succeed, then $E^{\prime}$ issues the query $\operatorname{send}(z, V)$ to session $z$.

If one of the checks fails, then session $z$ is aborted (i. e. $E^{\prime}$ aborts session $z$ ).

5. In case $E$ issues the test-session query to session $s^{*}, E^{\prime}$ issues the test-session query to session $s^{*}$ and returns the answer to $E$.

6. At the end of $E$ 's execution (after it has output its guess $b^{\prime}$ ), output $b^{\prime}$ as well.

Thus, it holds that $A d v_{E}^{\mathrm{SIG}(\Pi) \text {, Game } 3, O}(k) \leq A d v_{E^{\prime}}^{\Pi, \text { Game } 3, O}(k)$.

Finally,

$$
\begin{aligned}
A d v_{E}^{\mathrm{SIG}(\Pi)}(k) & \leq 2 P\left(\operatorname{Coll}_{\mathrm{SIG}(\Pi)}\right)+2 q_{s} N A d v_{M}^{\text {Sign }}(k)+q_{s} A d v_{E}^{\mathrm{SIG}(\Pi), \text { Game } 3, O}(k) \\
& \leq 2 P\left(\operatorname{Coll}_{\mathrm{SIG}(\Pi)}\right)+2 q_{s} N A d v_{M}^{\text {Sign }}(k)+q_{s} A d v_{E^{\prime}}^{I, \text { Game } 3, O}(k)
\end{aligned}
$$

Since by assumption protocol $\Pi$ is secure in $\mathrm{eCK}^{w}$, there is a negligible function $g$ such that $A d v_{E^{\prime}}^{I, \text { Game } 3, O}(k) \leq g(k)$ which completes the proof.

Remark 1. Let $\mathrm{M}^{w}$ and M-PFS be the security models obtained from eCK ${ }^{w}$ and eCK-PFS (respectively) by removing the ephemeral-key query and related restrictions in the freshness definitions. Then it can be shown in a similar way as above that for any $\mathrm{KE}$ protocol $\Pi \in \mathcal{D} \mathcal{H}-2$ secure in $\mathrm{M}^{w}$, the transformed protocol SIG $(\Pi)$ is secure in M-PFS using either a deterministic or a randomized SUF-CMA signature scheme.

Remark 2. In contrast to the SIG transformation, the MAC transformation $\mathcal{C}$ suggested in [6] applied to any protocol $\pi \in \mathcal{D H}-2$ does not yield a two-message key-exchange protocol secure in eCK-PFS since the transformed protocol is vulnerable to an attack that combines revealing the long-term secret keys of the actor of the test-session with revealing the long-term secret keys of the peer of the test-session after its completion. More precisely, an attacker can impersonate the peer of the test-session by first revealing the long-term secret keys of the actor (which allows him to create valid MACs on messages of his choice) and after the completion of the test-session revealing the long-term secret keys of the peer. For example, this attack shows that $\mathcal{C}(\mathrm{NAXOS})$ [6] is insecure in eCK-PFS. 


\section{$5 \quad$ NAXOS Revisited}

The NAXOS protocol [15, shown in Figure 3, provides an example of a protocol belonging to the class $\mathcal{D H}$-2, where $H_{1}:\{0,1\}^{*} \rightarrow \mathbb{Z}_{p}$ and $H_{2}:\{0,1\}^{*} \rightarrow\{0,1\}^{k}$ denote two hash functions and $r_{\hat{A}}, r_{\hat{B}} \in R\{0,1\}^{k}$. In analogy to Figure[1 note that $f_{\mathcal{I}}\left(r_{\hat{A}}, a, \Omega\right)=H_{1}\left(r_{\hat{A}}, a\right), f_{\mathcal{R}}\left(r_{\hat{B}}, b, \Omega\right)=H_{1}\left(r_{\hat{B}}, b\right), F_{\mathcal{I}}\left(f_{\mathcal{I}}\left(r_{\hat{A}}, a, \Omega\right), a, Y, \Omega\right)=$ $H_{2}\left(Y^{a}, B^{H_{1}\left(r_{\hat{A}}, a\right)}, Y^{H_{1}\left(r_{\hat{A}}, a\right)}, \hat{A}, \hat{B}\right)$, and $F_{\mathcal{R}}\left(f_{\mathcal{R}}\left(r_{\hat{B}}, b, \Omega\right), b, X, \Omega\right)=H_{2}\left(A^{H_{1}\left(r_{\hat{B}}, b\right)}\right.$, $\left.X^{b}, X^{H_{1}\left(r_{\hat{B}}, b\right)}, \hat{A}, \hat{B}\right)$.

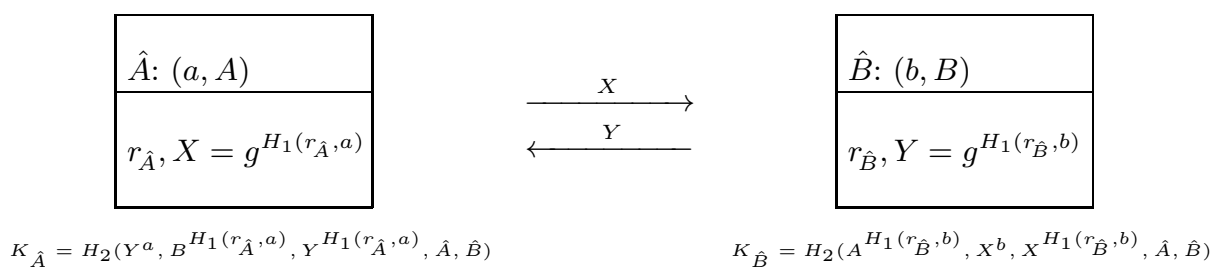

Fig. 3. NAXOS protocol [15]

The following proposition states that the NAXOS protocol is secure in $\mathrm{eCK}^{w}$. Proposition 1. Under the GAP-CDH assumption in the cyclic group $G$ of prime order $p$, NAXOS is secure in the $e C K^{w}$ model, when $H_{1}$ and $H_{2}$ are modeled as independent random oracles.

In contrast to the proof of NAXOS in the eCK model [15, the proof of Proposition 1 distinguishes between the cases whether or not an origin-session (instead of a matching session) exists for the test-session.

Proof (Sketch). Similar to [15]21, we analyze the following three events:

1. $D L \wedge K$

2. $T_{O} \wedge D L^{c} \wedge K$, and

3. $\left(T_{O}\right)^{c} \wedge D L^{c} \wedge K$, where

$T_{O}$ denotes the event that there exists an origin-session for the test-session, $D L$ denotes the event where there exists a party $\hat{C}$ such that the adversary $M$, during its execution, queries $H_{1}$ with $(*, c)$ before issuing a corrupt $(\hat{C})$ query and $K$ denotes the event that $M$ wins the security experiment against NAXOS by querying $H_{2}$ with $\left(\sigma_{1}, \sigma_{2}, \sigma_{3}, \hat{A}, \hat{B}\right)$, where $\sigma_{1}=C D H(Y, A), \sigma_{2}=C D H(B, X), \sigma_{3}=$ $C D H(X, Y)$ given that the test-session is $s^{*}$ with $T_{s^{*}}=(\hat{A}, \hat{B}, \mathcal{I}, X, Y)$.

Applying the SIG transformation on the NAXOS protocol yields the protocol SIG(NAXOS), depicted in Figure 4, Combining Proposition 11 with Theorem 1. we obtain the following result.

Corollary 1. Under the GAP-CDH assumption in the cyclic group $G$ of prime order p, using a deterministic SUF-CMA signature scheme, the SIG(NAXOS) protocol is secure in the eCK-PFS model, when $H_{1}, H_{2}$ are modeled as independent random oracles. 


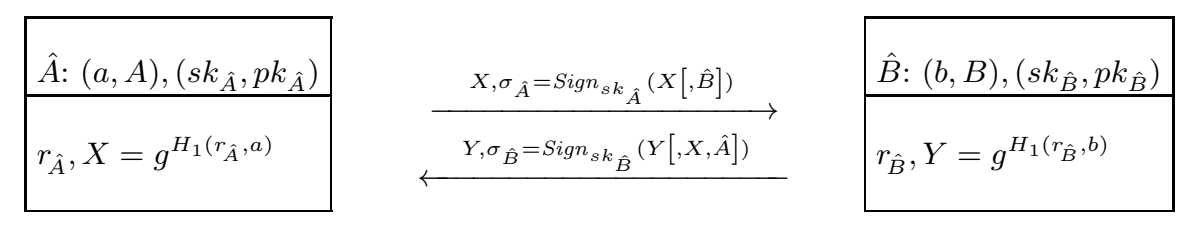

$$
\begin{aligned}
& K_{\hat{A}}=H_{2}\left(Y^{a}, B_{B}{ }^{H_{1}\left(r_{\hat{A}}, a\right)}, Y^{H_{1}\left(r_{\hat{A}}, a\right)}, \hat{A}, \hat{B}\right) \quad K_{\hat{B}}=H_{2}\left(A^{H_{1}\left(r_{\hat{B}}, b\right)}, X^{b}, X^{H_{1}\left(r_{\hat{B}}, b\right)}, \hat{A}, \hat{B}\right)
\end{aligned}
$$

Fig. 4. SIG(NAXOS) protocol

\section{Conclusions}

We provided two new eCK-like security notions, namely eCK ${ }^{w}$ and eCK-PFS. The $\mathrm{eCK}^{w}$ model slightly strengthens eCK by a more precise modeling of weakPFS. The stronger eCK-PFS notion guarantees PFS, even in the presence of eCK-like adversaries. Proving security in eCK-PFS provides strictly more guarantees than separately proving $\mathrm{eCK}^{w}$-security and PFS. Existing two-message KE protocols such as CMQV 21, NAXOS [15, or $\mathcal{C}(\mathrm{NAXOS})$ 6] fail to achieve security in eCK-PFS. We specified a security-strengthening transformation that transforms any two-message DH type KE protocol secure in $\mathrm{eCK}^{w}$ into a twomessage protocol secure in eCK-PFS. As future work, we would like to specify further transformations on KE protocols that are based on the newly developed security models in this work. It remains an open question whether there exist more efficient transformations that yield two-message KE protocols secure in eCK-PFS.

\section{References}

1. Bellare, M., Pointcheval, D., Rogaway, P.: Authenticated Key Exchange Secure against Dictionary Attacks. In: Preneel, B. (ed.) EUROCRYPT 2000. LNCS, vol. 1807, pp. 139-155. Springer, Heidelberg (2000)

2. Bellare, M., Rogaway, P.: Entity Authentication and Key Distribution. In: Stinson, D.R. (ed.) CRYPTO 1993. LNCS, vol. 773, pp. 232-249. Springer, Heidelberg (1994)

3. Bellare, M., Rogaway, P.: Provably secure session key distribution: the three party case. In: 27th Annual ACM Symposium on Theory of Computing, STOC 1995, pp. 57-66. ACM, New York (1995)

4. Boneh, D., Shen, E., Waters, B.: Strongly Unforgeable Signatures Based on Computational Diffie-Hellman. In: Yung, M., Dodis, Y., Kiayias, A., Malkin, T. (eds.) PKC 2006. LNCS, vol. 3958, pp. 229-240. Springer, Heidelberg (2006)

5. Boyd, C., Cliff, Y., Gonzalez Nieto, J.M., Paterson, K.G.: One-round key exchange in the standard model. Int. J. Applied Cryptography 1, 181-199 (2009)

6. Boyd, C., Nieto, J.G.: On Forward Secrecy in One-Round Key Exchange. In: Chen, L. (ed.) IMACC 2011. LNCS, vol. 7089, pp. 451-468. Springer, Heidelberg (2011) 
7. Canetti, R., Krawczyk, H.: Analysis of Key-Exchange Protocols and Their Use for Building Secure Channels. In: Pfitzmann, B. (ed.) EUROCRYPT 2001. LNCS, vol. 2045, pp. 453-474. Springer, Heidelberg (2001)

8. Cheng, Q., Ma, C., Hu, X.: A New Strongly Secure Authenticated Key Exchange Protocol. In: Park, J.H., Chen, H.-H., Atiquzzaman, M., Lee, C., Kim, T.-H., Yeo, S.-S. (eds.) ISA 2009. LNCS, vol. 5576, pp. 135-144. Springer, Heidelberg (2009)

9. Chow, S.S.M., Choo, K.-K.R.: Strongly-Secure Identity-Based Key Agreement and Anonymous Extension. In: Garay, J.A., Lenstra, A.K., Mambo, M., Peralta, R. (eds.) ISC 2007. LNCS, vol. 4779, pp. 203-220. Springer, Heidelberg (2007)

10. Dent, A.W.: A note on game-hopping proofs. Cryptology ePrint Archive, Report 2006/260 (2006), http://eprint.iacr.org/2006/260

11. Gennaro, R., Krawczyk, H., Rabin, T.: Okamoto-Tanaka Revisited: Fully Authenticated Diffie-Hellman with Minimal Overhead. In: Zhou, J., Yung, M. (eds.) ACNS 2010. LNCS, vol. 6123, pp. 309-328. Springer, Heidelberg (2010)

12. Katz, J., Lindell, Y.: Introduction to Modern Cryptography. Chapman Hall/CRC (2008)

13. Krawczyk, H.: HMQV: A High-Performance Secure Diffie-Hellman Protocol. In: Shoup, V. (ed.) CRYPTO 2005. LNCS, vol. 3621, pp. 546-566. Springer, Heidelberg (2005)

14. LaMacchia, B.A., Lauter, K., Mityagin, A.: Stronger security of authenticated key exchange. Cryptology ePrint Archive, Report 2006/073 (2006), http://eprint.iacr.org/

15. LaMacchia, B.A., Lauter, K., Mityagin, A.: Stronger Security of Authenticated Key Exchange. In: Susilo, W., Liu, J.K., Mu, Y. (eds.) ProvSec 2007. LNCS, vol. 4784, pp. 1-16. Springer, Heidelberg (2007)

16. Lee, J., Park, C.S.: An efficient authenticated key exchange protocol with a tight security reduction. Cryptology ePrint Archive, Report 2008/345 (2008), http://eprint.iacr.org/

17. Lee, J., Park, J.H.: Authenticated key exchange secure under the computational diffie-hellman assumption. Cryptology ePrint Archive, Report 2008/344 (2008), http://eprint.iacr.org/

18. Menezes, A., van Oorschot, P., Vanstone, S.: Handbook of Applied Cryptography (October 1996)

19. Okamoto, T., Pointcheval, D.: The Gap-problems: A New Class of Problems for the Security of Cryptographic Schemes. In: Kim, K.-C. (ed.) PKC 2001. LNCS, vol. 1992, pp. 104-118. Springer, Heidelberg (2001)

20. Shoup, V.: Sequences of games: a tool for taming complexity in security proofs. Cryptology ePrint Archive, Report 2004/332 (2006), http://eprint.iacr.org/

21. Ustaoglu, B.: Obtaining a secure and efficient key agreement protocol from (H)MQV and NAXOS. Designs, Codes and Cryptography 46(3), 329-342 (2008) 